Memoirs of the College of Science, University of Kyoto, Series A Vol. XXIX, Mathematics No. 1, 1955.

\title{
Basic theorems on general commutative rings
}

\author{
By
}

\author{
Masayoshi NAGATA \\ (Received August 10, 1954)
}

We want to group up some basic theorems in the general theory of commutative rings in the present note. Though most of results contained in the present note are not new, many of them will be sharpened, some of them will have simpler proofs and some of them will have more elementary proofs, than those which are already known.

Though the notions of local rings and of valuation rings are also basic for the theory of commutative rings, we will not observe them. Further, the normalization theorem (for finitely generated rings) is a basic theorem. But we will not discuss it. We concern mainly with the notion of rings of quotients, properties of integral dependence and the notion of rank of ideals.

In $\S 1$, we observe the notion of prime ideals. In $\S 2$, we study the notion of rings of quotients. In $\S 3$, we define the notion of prime divisors of ideals. In $\$ \S 4-5$, we study some properties of integral dependence. In $\$ 6$ we observe some properties of J-radicals of rings. In $\S 8$, we study the notion of rank of ideals. In $\S 9$, we observe some properties of normal Noetherian rings.

Terminology. If $\mathfrak{o}$ is a ring without identity, then we can imbed $\mathfrak{v}$ in a ring $\mathfrak{v}^{\prime}$ which has identity so that every ideal of $\mathfrak{v}$ is an ideal of $\mathfrak{v}^{\prime}$. Therefore the existence of the identity does not play essential rôle in general theory of rings, except for some extreme cases. Therefore we will assume the existence of the identity in any ring of consideration, unless the contrary is explicitly stated. Since we want to treat commutative rings, we assume also commutativity. A subring of a ring is assumed to have the same identity.

Results assumed to be well known. Besides some elementary notions and results on rings and fields, we need to know elementary properties of ideals and of Noetherian rings, which are con- 
tained in Waerden [11] Chapter 12.

$\S 1$. Prime ideals. (Cf. Krull [6], §1, No. 3)

We opserve first that the following conditions for ideals $p$ of a ring $\mathfrak{o}$ are equivalent to each other:

1) $\mathfrak{p}$ is a prime ideal of $\mathfrak{v}$. 2) If $a b$ is in $\mathfrak{p}(a, b \in \mathfrak{v})$, then either $a \in \mathfrak{p}$ or $b \in \mathfrak{p} .3$ ) If $\mathfrak{a}$ and $\mathfrak{b}$ are ideals of $\mathfrak{v}$ such that $\mathfrak{a b} \subseteq \mathfrak{p}$, then either $\mathfrak{a} \subseteq \mathfrak{p}$ or $\mathfrak{b} \subseteq \mathfrak{p}$. 4) If two ideals $\mathfrak{a}$ and $\mathfrak{b}$ contains $\mathfrak{p}$ properly, then $\mathfrak{a b}$ is not contained in $\mathfrak{p}$.

Let $\mathfrak{o}$ be a ring and let $S$ be a multiplicatively closed subset of $\mathfrak{p}$ which does not contain zero. An ideal $\mathfrak{p}$ of $\mathfrak{o}$ is called a maximal ideal with respect to $S$ if $\mathfrak{p}$ does not meet $S$ and if every ideal of $\mathfrak{o}$ properly containing $\mathfrak{p}$ meets $S$.

Proposition 1. Let $\mathfrak{o}$ be a ring and let $S$ be a multiplicatively closed subset of $\mathfrak{b}$. If an ideal $\mathfrak{a}$ of $\mathfrak{v}$ does not meet $S$, then there exists a maximal ideal $\mathfrak{p}$ of $\mathfrak{v}$ with respect to $S$ which contains $a$. Such $\mathfrak{p}$ is necessarily prime.

Proof. Existence follows from Zorn's lemma. Primeness of $p$ follows from 4) above.

If $\mathfrak{a}$ is an ideal of a ring $\mathfrak{v}$, then the intersection of all prime ideals of $\mathfrak{v}$ containing $\mathfrak{a}$ is called the radical of $\mathfrak{a}$; the radical of $\mathfrak{v}$ is the radical of the zero ideal of $\mathfrak{v}$.

Proposition 2. The radical $\mathfrak{a}^{\prime}$ of an ideal $\mathfrak{a}$ of a ring $\mathfrak{b}$ is the set of all elements of $\mathfrak{v}$ which are nilpotent modulo a.

Proof. Assume that an element $b$ is nilpotent modulo a. Then a power of $b$ is in every prime ideal containing $a$, whence $b$ is in every prime ideal containing a. Conversely, assume that an element $b$ is not nilpotent modulo $a$. Then the multiplicatively closed set $\left\{b^{n} ; n=1, \cdots, m, \cdots\right\}$ does not meet $a$, whence there exists a prime ideal $\mathfrak{p}$ containing $a$ which does not contain $b$ by Proposition 1.

Corollary. An ideal a of a ring $\mathfrak{o}$ is semi-prime if and only if $\mathfrak{o} / \mathfrak{a}$ has no nilpotent element. (We call an ideal a semi-prime ideal if it is an intersection of prime ideals.)

When $\mathfrak{a}$ is an ideal of a ring $\mathfrak{v}$, a prime ideal $\mathfrak{p}$ of $\mathfrak{o}$ is called a minimal prime divisor of $a$ if it is minimal among prime ideals containing a.

Proposition 3. If an ideal $\mathfrak{a}$ of $a$ ring $\mathfrak{o}$ is contained in $a$ prime ideal $\mathfrak{p}$, then $\mathfrak{p}$ contains a minimal prime divisor of $a$.

The proof is easy by virtue of Zorn's lemma. 
Corollary. The radical of an ideal a is the intersection of all minimal prime divisors of a.

Proposition 4. If $\mathfrak{p}_{1}, \cdots, \mathfrak{p}_{n}$ are prime ideals of a ring $\mathrm{o}$ and if $\mathfrak{a}$ is an ideal of $\mathfrak{o}$ which is not contained in any of $\mathfrak{p}_{1}, \cdots, \mathfrak{p}_{n}$, then there exists an element $a$ of a which is not contained in any of $p_{1}, \cdots, p_{n}$.

Proof. If one of $\mathfrak{p}_{i}$, say $\mathfrak{p}_{n}$, is contained in some other $\mathfrak{p}_{j}$, then we may omit $\mathfrak{p}_{n}$ without loss of generality. Therefore we may assume that there is no inclusion relation among $\mathfrak{p}_{1}, \cdots, \mathfrak{p}_{n}$. Then there exists an element $a_{i}$ of $\mathfrak{p}_{i} \cdots \mathfrak{p}_{i-1} \mathfrak{p}_{i+1} \cdots \mathfrak{p}_{n} \mathfrak{a}$ which is not in $\mathfrak{p}_{i}$ for each $i$ (see 3) above). Then $a=a_{1}+\cdots+a_{n}$ is in $a$ and is in none of $\mathfrak{p}_{i}$.

Remark. If $\mathfrak{q}$ is a primary ideal of a ring $\mathfrak{o}$ and if $\mathfrak{v}^{\prime}$ is a subring of $\mathfrak{o}$, then $\mathfrak{q} \cap \mathfrak{o}^{\prime}$ is a primary ideal of $\mathfrak{v}^{\prime}$. If $\mathfrak{q}$ is a prime ideal, then $\mathfrak{q} \cap \mathfrak{v}^{\prime}$ is prime. [10])

$\S$ 2. Rings of quotients. (Cf. Krull [6], Chevalley [3], Uzkov

Let $\mathfrak{o}$ be a ring and let $U$ be the set of non-zero-divisors in o. In the set $P=\{(a, u) ; a \in \mathfrak{v}, u \in U\}$ we introduce a equivalent relation, namely, $(a, u)$ is equivalent to $(b, v)$ if and only if $a v=$ $b u$. We denote the class of $(a, u)$ by $a / u$. The set $Q$ of the equivalent classes becomes a ring under operations $(a / u)+(b / v)$ $=(a v+b u) / u v,(a / u)(b / v),=a b / u v . \quad Q$ is called the total quotient ring of $\mathfrak{o}$; here $a / 1$ is identified with $a$ in $\mathfrak{o} . \quad Q$ contains $\mathfrak{o}$ as a subring by this identification and $Q$ is generated by $\mathfrak{o}$ and inverses of elements of $U$ (every element of $U$ has inverse in $Q$ ).

Now let $S$ be a multiplicatively closed subset of $\mathfrak{o}$ which does not contain zero. When $S$ contains no zero-divisor, then the subring of $Q$ generated by $\mathfrak{v}$ and inverses of elements of $S$ is called the ring of quotients of o with respect to $S$. In general case, set $\mathfrak{n}=\{a ; a \in \mathfrak{v}, a s=0$ for some $s \in S\}$. Then $\mathfrak{n}$ is an ideal of $\mathfrak{v}$. Let $\phi$ be the natural homomorphism from $\mathfrak{o}$ onto $\mathfrak{v} / \mathfrak{n t}$. Then $\phi(S)$ is multiplicatively closed; this set does not contain any zerodivisors. For, if $\phi(s) \phi(a)=0 \quad(s \in S, a \in \mathfrak{p})$, then $s a \in \mathfrak{n}$ and therefore there exists an $s^{\prime}$ of $S$ such that $a s s^{\prime}=0$. Since $s s^{\prime}$ is in $S$, we see that $a$ is in $n$ and therefore $\phi(a)=0$, which proves that no element of $\phi(S)$ is a 7ero-divisor.*) Therefore we can construct

* Observe that if $a \in U$ then $\phi(a)$ is not a zero-divisor, as is easily seen by the same proof as here. 
the ring of quotients of $\phi(\mathfrak{v})$ with respect to $\phi(S)$; this ring will be called the ring of quotients of $\mathfrak{v}$ with respect to $S$ and will be denoted by $\mathfrak{o}_{s}$. (Observe that if $S$ contains no zero-divisor, then $\mathfrak{\imath}=0$, whence $\phi$ is the identity mapping. Therefore the previous definition is contained in this case.)

When $S$ is the complementary set of a prime ideal $\mathfrak{p}$ of $\mathfrak{o}$, then $\mathfrak{v}_{s}$ is called the ring of quotients of $\mathfrak{v}$ with respect to $\mathfrak{p}$ and is denoted by $\mathfrak{o}_{\mathfrak{p}}$.

A ring $\mathfrak{b}^{\prime}$ is called a ring of quotients of the ring $\mathfrak{o}$ if there exists a multiplicatively closed subset $S$ of $\mathfrak{v}$ which does not contain zero such that $\mathfrak{b}^{\prime}=\mathfrak{o}_{\mathrm{s}}$.

We use the following notations :

1) When $\mathfrak{a}$ is a subset of the ring $\mathfrak{o}$, we denote by $\mathrm{ao}_{s}$ the ideal $\phi(\mathfrak{a}) \mathfrak{o}_{s}$.

2) When $\mathfrak{a}^{\prime}$ is an ideal of $\mathfrak{o}_{s}$, we denote by $\mathfrak{a}^{\prime} \cap \mathfrak{o}$ the ideal $\phi^{--}\left(\mathfrak{a}^{\prime} \cap \phi(\mathfrak{o})\right)$.

Remark. If $\mathfrak{a}^{\prime}$ and $\mathfrak{b}^{\prime}$ are ideals of $\mathfrak{o}_{s}$, then $\left(\mathfrak{a}^{\prime} \cap \mathfrak{b}^{\prime}\right) \cap \mathfrak{v}=$ $\left(\mathfrak{a}^{\prime} \cap \mathfrak{v}\right) \cap\left(\mathfrak{b}^{\prime} \cap \mathfrak{v}\right)$.

Assume that there exists a ring $\mathfrak{v}^{\prime}$ as follows: 1) There exists a homomorphism $\pi$ from $\mathfrak{v}$ into $\mathfrak{v}^{\prime}$ and 2) every element of $\pi(S)$ has inverse in $\mathfrak{v}^{\prime}$. Then from $\pi(s) \pi(a)=0(s \in S, a \in \mathfrak{v})$ it follows that $\pi(a)=0$. Therefore the kernel of $\phi$ is contained in that of $\pi$. Therefore there exists a homomorphism from $\mathfrak{o}_{s}$ onto the subring of $\mathfrak{b}^{\prime}$ generated by $\pi(\mathfrak{b})$ and the inverses of elements of $\pi(S)$. Thus we see that $\mathfrak{o}_{S}$ is the most " universal" ring in which every element of $S$ is mapped (under homomorphism) to unit. Therefore

Proposition 1. Let $\mathfrak{a}$ be an ideal of $\mathfrak{o}$ which does not meet $S$. Let $\theta$ be the natural homomorphism from $\mathfrak{v}$ onto $\mathfrak{o} / \mathfrak{a}$. Then $\theta\left(\mathfrak{o}_{s}\right)$ $=\mathfrak{o}_{s} / \mathfrak{a n}_{s}$.

Next we observe correspondence between ideals of $\mathfrak{o}$ and those of $\mathfrak{o}_{s}$.

Proposition 2. If $\mathfrak{a}^{\prime}$ is an ideal of $\mathfrak{o}_{s}$, then $\left(\mathfrak{a}^{\prime} \cap \mathfrak{o}\right) \mathfrak{o}_{s}=\mathfrak{a}^{\prime}$.

Proof. We have $\left(\mathfrak{a}^{\prime} \cap \mathfrak{o}\right) \mathfrak{o}_{s}=\phi\left(\phi^{-1}\left(\mathfrak{a}^{\prime} \cap \phi(\mathfrak{b})\right) \mathfrak{o}_{s}=\left(\mathfrak{a}^{\prime} \cap \phi(\mathfrak{b})\right) \mathfrak{o}_{s} \subseteq \mathfrak{a}^{\prime}\right.$. Assume that $\phi(a) / \phi(s) \in \mathfrak{a}^{\prime}(a \in \mathfrak{p}, s \in S)$. Then $\phi(a)=\phi(s)(\phi(a) / \phi(s))$ is in $\mathfrak{a}^{\prime} \cap \phi(\mathfrak{v})$. Since $1 / \phi(s)$ is in $\mathfrak{o}_{s}$, we have $\phi(a) / \phi(s)$ is in $\left(a^{\prime} \cap \mathfrak{v}\right) \mathfrak{o}_{s}$.

Corollary. If $\mathfrak{v}$ is Noetherian, then $\mathfrak{o}_{s}^{-}$is Noetherian.

Proposition 3. Assume that $\mathfrak{q}$ is a primary ideal of $\mathfrak{o}$ belong- 
ing to a prime ideal $\mathfrak{p}$. Then: 1) if $\mathfrak{p}$ meets $S$, then $\mathfrak{p o}_{S}=\mathfrak{q o}_{S}=\mathfrak{o}_{S}$; 2) if $\mathfrak{p}$ does not meet $S$, then $\mathfrak{q}$ contains $\mathfrak{n}, \mathfrak{p o}_{S}$ is a prime ideal, $q \mathrm{v}_{s}$ is a primary ideal belonging to $\mathfrak{p o}_{S}, \mathfrak{p o}_{s} \cap \mathfrak{v}=\mathfrak{p}$ and $\mathfrak{q o}_{s} \cap \mathfrak{v}=\mathfrak{q}$.

Proof. If $\mathfrak{p}$ meets $S$, then $\mathfrak{q}$ meets $S$ because any power of an element of $S$ is in $S$. Therefore in this case we have $\mathbf{p o s}_{S}=$ $\mathfrak{q b}_{S}=\mathfrak{p}_{s}$. Assume that $\mathfrak{p}$ does not meet $S$. If $a$ is in $\mathfrak{n}$, then there exists an $s$ in $S$ such that $a s=0$. Since $s$ is not in $\mathfrak{p}$, we have $a$ is in $\mathfrak{q}$, which proves that $\mathfrak{q}$ contains $\mathfrak{n}$. Let $b$ be an element of $\mathfrak{q 0}_{s} \cap \mathfrak{v}$. Then $\phi(b)=\phi(q) / \phi(s)$ with $q \epsilon \mathfrak{q}, s \in S$. Therefore $\phi(b s)$ is in $\phi(\mathfrak{q})$. Since $\mathfrak{q}$ contains $\mathfrak{n}, b s$ is in $\mathfrak{q}$. Since $s$ is not in $\mathfrak{p}, b$ is in $\mathfrak{q}$, which shows that $\mathfrak{q} \supseteq \mathfrak{q}_{s} \cap \mathfrak{v}$. Since the converse inclusion is evident, we have $\mathfrak{q}=\mathfrak{q}_{\mathfrak{s}} \cap \mathfrak{v}$. Since $\mathfrak{p}$ is also a primary ideal, we see also that $\mathfrak{p}=\mathfrak{p o}_{s} \cap \mathfrak{o}$. Next we assume that $\phi(a) / \phi(s)$ is not in $\mathfrak{q o}_{s}$ and that $\phi(a b) / \phi(s t)$ is in $\mathfrak{q o}_{s}$, where $a, b \in \mathfrak{D}$ and $s, t \in S$. Then by above observation, $a$ is not in $\mathfrak{q}$ and $a b$ is in $q$. Therefore there exists a natural number $r$ such that $b^{r}$ is in $\mathfrak{q}$, whence

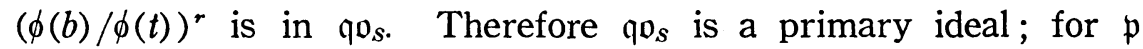
the same holds with $r=1$ and therefore $\mathrm{po}_{s}$ is a prime ideal. Since every element of $\mathfrak{p}$ is nilpotent modulo $\mathfrak{q}$, every element of $\mathfrak{p o}_{s}$ is nilpotent modulo $\mathfrak{q} \mathfrak{o}_{s}$, whence $\mathfrak{q} \mathfrak{o}_{s}$ is a primary ideal belonging to $\mathrm{po}_{s}$. Thus the proof is completed.

Corollary 1. Let $\mathfrak{p}$ be a prime ideal of $\mathfrak{o}$. Then $\mathrm{po}_{s}$ is $a$ maximal ideal of $\mathfrak{o}_{S}$ if and only if $\mathrm{p}$ is a maximal ideal with respect to $S$.

Corollary 2. If an ideal $\mathfrak{v}$ of $\mathfrak{v}$ does not meet $S$, then $\mathfrak{v o} \neq \mathfrak{o}_{s}$.

Proposition 4. Let $\mathfrak{q}_{1}, \cdots, \mathfrak{q}_{n}$ be primary ideals of $\mathfrak{0}$. Then $\left(\mathfrak{q}_{1} \cap \cdots \cap \mathfrak{q}_{n}\right) \mathfrak{o}_{s}=\mathfrak{q}_{1} \mathfrak{o}_{S} \cap \cdots \cap \mathfrak{q}_{n} \mathfrak{o}_{s}$. If $\mathfrak{q}_{1} \neq \underset{i \geq 2}{\cap} \mathfrak{q}_{i}$ and if $\mathfrak{q}_{1} \mathfrak{o}_{S} \neq \mathfrak{o}_{s}$, then $\mathfrak{q}_{1} \mathfrak{o}_{s} \notin \underset{P \geq 2}{\cap} \mathfrak{q}_{i} \mathfrak{o}_{s}$

Proof. Set $a=q_{1} \cap \cdots \cap \mathfrak{q}_{n}$. We renumber $\mathfrak{q}_{r}$ so that $q \cap S$ is empty if and only if $i \leq r$. Since $\mathfrak{q}_{i} \mathfrak{o}_{s}$ contains $\mathfrak{a v}_{s}, \mathfrak{a v}_{S}$ is contained in $\mathfrak{q}_{1} \mathfrak{v}_{S} \cap \cdots \cap \mathfrak{q}_{n} \mathfrak{o}_{S}\left(=\mathfrak{q}_{1} \mathfrak{o}_{S} \cap \cdots \cap \mathfrak{q}_{1} \mathfrak{o}_{S}\right)$. Let $\phi(a) / \phi(s) \quad(a \in \mathfrak{D}, s \in S)$ be an element of $\mathfrak{q}_{1} \mathfrak{D}_{3} \cap \cdots \cap \mathfrak{q}_{,} \mathfrak{b}_{s}$. Since $\mathfrak{q}_{i} \mathfrak{o}_{s} \cap \mathfrak{v}=\mathfrak{q}_{i}$ for $i \leq r, a$ is in $\mathfrak{q}_{1} \cap \cdots \cap$ $\mathfrak{q}_{r}$. Take elements $s_{r+1}, \cdots, s_{n}$ of $S$ so that $s_{r+j}$ is in $\mathfrak{q}_{r+j}$. Then $a^{\prime}=$ $a s_{r+1} \cdots s_{n}$ is in $\mathfrak{a}$. Therefore $\phi(\mathfrak{a}) / \phi(s)=\phi\left(a^{\prime}\right) / \phi\left(s s_{r+1} \cdots s_{n}\right)$ is in $\mathfrak{a b _ { s }}$, which proves the converse inclusion. Thus we see that $\mathfrak{a b}_{s}=\mathfrak{q}_{1} \mathfrak{o}_{s}$ $\cap \cdots \cap \mathfrak{q}_{1} \mathfrak{o}_{s}$. Now we assume that $\mathfrak{q}_{1} \notin \cap_{i \geq 2} \mathfrak{q}_{i}$ and that $r \geq 1$. Take an element $a$ of $\underset{i \geq 2}{\cap \mathfrak{q}_{r}}$ which is not in $\mathfrak{q}_{1}$. Then since $\mathfrak{q}_{1} \mathfrak{o}_{s} \cap \mathfrak{v}=\mathfrak{q}_{1}$, $\phi(a)$ is not in $\mathfrak{q}_{1} \mathfrak{o}_{s}$, which shows that $\mathfrak{q}_{1} \mathfrak{v}_{s} \notin \underset{i \geq 2}{\mathfrak{n}_{1} \mathfrak{o}_{s}}$.

Corollary. Assume that the zero ideal of $\mathfrak{0}$ can be expressed 
as the intersection of primary ideals $\mathfrak{q}_{1}, \cdots, \mathfrak{q}_{n}$ of $\mathfrak{o}$, where $\mathfrak{q}_{i} \cap S$ is empty if and only if $i \leq r$. Then the ideal $\mathfrak{n}\left(=\phi^{-1}(0)\right)$ coincides with $\mathfrak{q}_{1} \cap \cdots \cap q_{r}$.

Proof. $\mathfrak{n}=\phi^{-1}(0)$ and therefore $\mathfrak{n}=(0) \mathfrak{v}_{s} \cap \mathfrak{v}$. By our assumption, (0) $\mathfrak{o}_{s}=\mathfrak{q}_{1} \mathfrak{b}_{s} \cap \cdots \cap \mathfrak{q}_{r} \mathfrak{o}_{s}$ and therefore $\mathfrak{n}=\mathfrak{q}_{1} \cap \cdots \cap \mathfrak{q}_{r}$.

Proposition 5. Let $\mathrm{o}$ be a ring and let $S$ be a multiplicatively closed subset of $\mathfrak{v}$ which does not contain zero. Let $S^{\prime}$ be a multiplicatively closed subset of $\mathfrak{o}_{S}$ which does not contain zero. Let $S^{\prime \prime}$ be the multiplicatively closed subset of $\mathfrak{v}$ generated by $S$ and all elements $s^{\prime \prime}$ of $\mathfrak{v}$ such that with a suitable element $s$ of $S, \phi\left(s^{\prime \prime}\right) / \phi(s)$ is in $S^{\prime}$, where $\phi$ is the natural homomorphism from $\mathfrak{o}$ into $\mathfrak{v}_{s}$. Then $\mathfrak{o}_{S^{\prime \prime}}=\left(\mathfrak{o}_{S}\right)_{S^{\prime}}$.

Proof. Let $\theta$ and $\pi$ be the natural homomorphisms from $\mathfrak{D}$ into $\mathfrak{o}_{s^{\prime \prime}}$ and from $\mathfrak{o}_{S}$ into $\left(\mathfrak{o}_{S}\right)_{s^{\prime}}$ respectively. Then every element of $\pi \phi\left(S^{\prime \prime}\right)$ has inverse in $\left(\mathfrak{v}_{s}\right)_{S^{\prime}}$ and $\left(\mathfrak{o}_{s}\right)_{S^{\prime}}$ is generated by $\pi \phi(\mathfrak{o})$ and inverses of elements of $\pi \phi\left(S^{\prime \prime}\right)$. Therefore there exists a homomorphism from $\mathfrak{D}_{s^{\prime \prime}}$ onto $\left(\mathfrak{o}_{s}\right)_{s^{\prime}}$. Let $\mathfrak{n}^{\prime \prime}$ be the kernel of $\pi \phi$. Then for every element $a$ of $\mathfrak{n}^{\prime \prime}$, there exists an element $\phi\left(s^{\prime \prime}\right) / \phi(s)$ $\left(s \in S, s^{\prime \prime} \epsilon S^{\prime \prime}\right)$ of $S^{\prime}$ such that $\phi(a) \phi\left(s^{\prime \prime}\right) / \phi(s)=0$. Then $a s^{\prime \prime}$ is in the kernel of $\phi$, whence there exists an element $s^{\prime}$ of $S$ such that $a s^{\prime \prime} s^{\prime}=0$. Since $s^{\prime \prime} s^{\prime}$ is in $S^{\prime \prime}, a$ is in the kernel of $\theta$. Therefore $\theta=\pi \phi$, whence $\mathfrak{o}_{s^{\prime \prime}}=\left(\mathfrak{o}_{s}\right)_{s^{\prime}}$.

Proposition 6. Let $S$ be a multiplicatively closed subset of a ring $\mathfrak{D}$ which does not contain zero. Let $\left\{\mathfrak{p}_{\lambda} ; \lambda \in \wedge\right\}$ be the set of maximal ideals of $\mathfrak{D}$ wih respect to $S$ and let $S^{\prime}$ be the intersection of the complementary sets of ideals $\mathfrak{p}_{\lambda}$ with respect to $\mathfrak{v}$ (for all $\lambda \in \wedge)$. Then $\mathfrak{o}_{s}=\mathfrak{o}_{s^{\prime}}$.

Proof. Let $\phi$ be the natural homomorphism from $\mathfrak{o}$ into $\mathfrak{o}_{s}$. Then every element of $\phi\left(S^{\prime}\right)$ is a unit in $\mathfrak{o}_{s}$ by Corollary 1 to Proposition 3. Therefore our assertion follows from Proposition 1.

Proposition 7. Let $\mathrm{v}$ be a ring and let $S$ be a multiplicatively closed subset of $\mathfrak{v}$ which contains no zero-divisor. If a ring $\mathfrak{b}^{\prime}$ contains $\mathfrak{o}$ and is contained in $\mathfrak{o}_{s}$, then $\mathfrak{o}_{s}=\mathfrak{v}^{\prime}$.

The proof is easy by the definition of rings of quotients.

$\S 3$. Prime divisors.

Let $\mathfrak{a}$ be an ideal of a ring $\mathfrak{o}$ and let $S$ be the set of elements of $\mathfrak{v}$ which are not zero-divisors modulo a. Then $S$ is multiplicatively closed and does not meet a. A prime ideal $\mathfrak{p}$ is called a maximal prime divisor of $a$ if it is maximal with respect to $S$ and 
if it contains a. A prime ideal $\mathfrak{q}$ of $\mathfrak{b}$ is called a prime divisor of $\mathfrak{a}$ if there exists a multiplicatively closed subset $S^{\prime}$ of $\mathfrak{o}$ which does not meet a such that $\mathrm{qo}_{s^{\prime}}$ is a maximal prime divisor of $\mathfrak{a b}_{S^{\prime}}$. (By Proposition 3 in $\S 2$, such $\mathfrak{q}$ contains a.)

The followings can be proved easily:

(1) Any maximal prime divisor is a prime divisor. Further any prime divisor is contained in a maximal prime divisor.

(2) A minimal prime divisor is a prime divisor; it is minimal among prime divisors. Further, if $\mathfrak{p}$ is a minimal prime divisor of an ideal $\mathfrak{a}$ of a ring $\mathfrak{o}$, then $\mathfrak{a o p}_{\mathfrak{p}}$ is a primary ideal belonging to $\mathfrak{p o p}_{\mathfrak{p}}$. And therefore $\mathfrak{a v}_{\mathfrak{p}} \cap \mathfrak{v}$ is a primary ideal belonging to $\mathfrak{p}$; this ideal is called the primary component of $\mathfrak{a}$ belonging to $\mathfrak{p}$.

(3) An ideal is primary if and only if it has only one prime divisor.

(4) Assume that an ideal $\mathfrak{a}$ of a ring $\mathfrak{o}$ is the intersection of primary ideals $\mathfrak{q}_{1}, \cdots, \mathfrak{q}_{n}$ of $\mathfrak{b}$. If the intersection $\mathfrak{q}_{1} \cap \cdots \cap \mathfrak{q}_{n}$ is

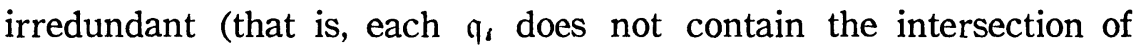
other $\mathfrak{q}_{\mathfrak{j}}$ ), then the set of prime divisors of $\mathfrak{a}$ is the set of prime divisors of $q_{1}, \cdots, q_{n}$.

A prime divisor which is not minimal is called an imbedded prime divisor.

§4. Integral dependence.

We say that an element $a$ of a ring $\mathfrak{o}^{\prime}$ is integral over its subring $\mathfrak{o}$ if there exist elements $c_{1}, \cdots, c_{n}$ of $\mathfrak{o}$ such that $a^{n}+c_{1} a^{n-1}$ $+\cdots+a_{n}=0$, i. e., $a$ is a root of a monic polynomial over $\mathfrak{o}$. We say that $\mathfrak{v}^{\prime}$ is integral over $\mathfrak{v}$ if every element of $\mathfrak{v}^{\prime}$ is integral over $\mathfrak{v}$.

Lemma 1. If a ring $\mathfrak{o}^{\prime}$ is integral over its subring $\mathfrak{o}$ and if $\phi$ is a homomorphism (from $\mathfrak{o}^{\prime}$ into some ring), then $\phi\left(\mathfrak{o}^{\prime}\right)$ is integral over $\phi(\mathfrak{v})$.

This follows immediately from the definition of integral dependence.

Lemma 2. Assume that a ring $\mathfrak{v}^{\prime}$ is integral over its subring v. If $S$ is a multiplicatively closed subset of $\mathfrak{D}$ which does not contain zero, then $\mathfrak{v}_{s}^{\prime}$ is integral over $\mathfrak{o}_{s}$.

Proof. Let $\phi$ be the natural homomorphism from $\mathfrak{b}^{\prime}$ into $\mathfrak{b}^{\prime} s$. Then since $S$ is a subset of $\mathfrak{b}, \phi$ is the natural homomorphism from $\mathfrak{o}$ into $\mathfrak{o}_{s}$ if it is restricted to $\mathfrak{o}$. Let $\phi(a) / \phi(s)\left(a \in \mathfrak{o}^{\prime}, s \in S\right)$ be an element of $\mathfrak{o}^{\prime} s$. Then $\phi(a)$ is integral over $\phi(\mathfrak{v})$ and $\phi(s)$ is unit in $\mathfrak{o}_{s}$. Therefore $\phi(a) / \phi(s)$ is integral over $\mathfrak{o}_{s}$. 
Lemma 3. If a field $K$ is integral over its subring $I$, then $I$ is a field. (Krull [7]; cf. [4])

Proof. Let $a$ be an element of $I$ which is not zero. Since $a^{-1}$ is in $K, a^{-1}$ is integral over $I$, whence there exist elements $c_{1}, \cdots, c_{n}$ of $I$ such that $\left(a^{-1}\right)^{n}+c_{1}\left(a^{-1}\right)^{n+1}+\cdots+c_{n}=0$. Then $a^{-1}=$ $-\left(c_{1}+c_{2} a+\cdots+c_{n} a^{n-1}\right)$, which is in $I$. Therefore $I$ is a field.

Lemma 4. If an integrity domain $\mathfrak{o}$ is integral over its subfield $K$, then $\mathfrak{v}$ is a field.

Proof. Let $a$ be an element of $\mathfrak{v}$ which is not zero. Then there exist elements $c_{1}, \cdots, c_{n}$ of $K$ such that $a^{n}+c_{1} a^{n-1}+\cdots+c_{n}=0$. Since $\mathfrak{v}$ is an integrity domain, we may assume that $c_{n} \neq 0$. Then $a^{-1}=-c_{n}^{-1}\left(a^{n-1}+c_{1} a^{n-2}+\cdots+c_{n-1}\right)$, which is in $\mathfrak{v}$. Therefore $\mathfrak{v}$ is a field.

Proposition 1. Assume that a ring $\mathfrak{b}^{\prime}$ is integral over its subring $\mathfrak{o}$. Let $\mathfrak{p}$ be a prime ideal of $\mathfrak{o}$ and let $S$ be the complementary set of $\mathfrak{p}$ with respect to $\mathfrak{o}$. Then a prime ideal $\mathfrak{p}^{\prime}$ of $\mathfrak{o}^{\prime}$ lies over $\mathfrak{p}$ (that is, $\mathfrak{p}^{\prime} \cap \mathfrak{o}=\mathfrak{p}$ ) if and only if $\mathfrak{p}^{\prime}$ is a maximal ideal with respect to $S$. (Krull [7] and Cohen-Seidenberg [4])

Proof. Assume first that a prime ideal $\mathfrak{p}^{\prime}$ of $\mathfrak{d}^{\prime}$ lies over $\mathfrak{p}$. Then since $\mathfrak{p}^{\prime} \cap \mathfrak{v}=\mathfrak{p}, \mathfrak{p}^{\prime}$ does not meet $S$. Therefore $\mathfrak{p}^{\prime} \mathfrak{o}^{\prime}{ }_{S}$ is different from $\mathfrak{o}^{\prime}{ }_{s}$ and $\mathfrak{p}^{\prime} \mathfrak{v}^{\prime}{ }_{s} \cap \mathfrak{o}_{s}=\mathfrak{p o}_{s}$. By Lemmas 1 and $2, \mathfrak{o}^{\prime}{ }_{s} / \mathfrak{p}^{\prime} \mathfrak{v}^{\prime}$ is integral over $\mathfrak{o}_{S} / \mathfrak{p o}_{s}$ (which is a field). Therefore $\mathfrak{o}^{\prime}{ }_{S} / \mathfrak{p}^{\prime} \mathfrak{v}^{\prime}{ }_{s}$ is a field by Lemma 4, which shows that $\mathfrak{p}^{\prime} \mathfrak{v}^{\prime}{ }_{S}$ is maximal. Therefore $\mathfrak{p}^{\prime}$ is a maximal ideal with respect to $S$ by Corollary 1 to Proposition 3 in $\$ 2$. Conversely, assume that $\mathfrak{p}^{\prime}$ is a maximal ideal with respect to $S$. Then $\mathfrak{p}^{\prime} \mathfrak{v}_{S}{ }_{S}$ is maximal. Therefore by Lemma $3, \mathfrak{p}^{\prime} \mathfrak{o}_{S}^{\prime} \cap \mathfrak{o}_{s}$ is maximal, whence it is $\mathfrak{p o}_{s}$. Thus we see that $\mathfrak{p}^{\prime} \cap \mathfrak{v}$ $=\mathfrak{p}$.

Corollary 1. For any prime ideal $\mathfrak{p}$ of $\mathfrak{o}$, there exist prime ideals of $\mathfrak{o}^{\prime}$ which lie over $\mathfrak{p}$; there is no inclusion relation among prime ideals of $\mathfrak{v}^{\prime}$ which lie over the same prime ideal of $\mathfrak{o}$.

Corollary 2. If $\mathfrak{p}_{1} \subset \cdots \subset \mathfrak{p}_{n}$ is an ascending chain of prime ideals $\mathfrak{p}_{i}$ of $\mathfrak{v}$, then there exist prime ideals $\mathfrak{p}_{1}^{\prime}, \cdots, \mathfrak{p}_{n}{ }_{n}$ of $\mathfrak{o}^{\prime}$ such that 1) $\mathfrak{p}_{i}^{\prime} \cap \mathfrak{v}=\mathfrak{p}_{i}$ for each 1 and 2) $\mathfrak{p}^{\prime} \subset \cdots \subset \mathfrak{p}_{n}^{\prime}$; here, if there is no prime ideal $\mathfrak{p}$ such that $\mathfrak{p}_{i} \subset \mathfrak{p} \subset \mathfrak{p}_{i+1}$, then there is no prime ideal $\mathfrak{p}^{\prime}$ of $\mathfrak{o}^{\prime}$ such that $\mathfrak{p}_{i}^{\prime} \subset \mathfrak{p}^{\prime} \subset \mathfrak{p}^{\prime}{ }_{i+1}$.

Proof. Existence is easy by induction on $n$, while the latter follows from Corollary 1.

Proposition 2. If a ring $\mathfrak{v}^{\prime}$ is a finite module over its subring 
$\mathfrak{b}$, then $\mathfrak{o}^{\prime}$ is integral over $\mathfrak{o} . \quad$ (Nagata [9])

Proof. Take elements $u_{1}=1, u_{2}, \cdots, u_{n}$ of $\mathfrak{v}^{\prime}$ such that $\mathfrak{o}^{\prime}=\sum_{j} \mathrm{o} u_{j}$. Then for every element $a$ of $\mathfrak{v}^{\prime}$, we can write that $a u_{i}=\sum_{j} a_{i j} u_{j}$ $\left(a_{i j} \in \mathfrak{p}\right)$. Then we see that the determinant $\left|\left(\delta_{i j} a-a_{i j}\right)\right|=0$ (where $\delta_{i j}$ denotes Kronecker's $\delta$ ) because $u_{1}=1$. Therefore $a$ is integral over $\mathbf{D}$.

Remark. Observe, conversely, that if an element $a$ is integral over $\mathfrak{o}$, then $\mathfrak{o}[a]$ is a finite $\mathfrak{o}$-module, because $a$ satisfies a monic equation.

Corollary 1. Let $\mathfrak{o}$ be a subring of a ring $\mathfrak{o}^{\prime}$. Then the set $\mathfrak{v}^{\prime \prime}$ of elements of $\mathfrak{v}^{\prime}$ which are integral over $\mathfrak{o}$ form a subring of $\mathfrak{b}^{\prime}$.

This $\mathfrak{v}^{\prime \prime}$ is called the integral closure of $\mathfrak{v}$ in $\mathfrak{v}^{\prime}$. If $\mathfrak{v}=\mathfrak{v}^{\prime \prime}$, then we say that $\mathfrak{o}$ is integrally closed in $\mathfrak{v}^{\prime}$.

Proof. Let $a$ and $b$ be elements of $\mathfrak{v}^{\prime \prime}$. Then $\mathfrak{o}[a, b]$ is a finite $\mathfrak{o}$-module and is a ring. Therefore $\mathfrak{v}[a, b]$ is integral over $\mathfrak{0}$; in particular, $a-b$ and $a b$ are in $\mathfrak{v}^{\prime \prime}$. Therefore $\mathfrak{v}^{\prime \prime}$ is a ring.

Corollary 2. If an element $a$ is integral over a ring $\mathrm{o}^{\prime}$ and if $\mathfrak{v}^{\prime}$ is integral over its subring $\mathfrak{v}$, then $a$ is integral over $\mathfrak{v}$.

Corollary 3. Let $\mathrm{o}$ be a Noetherian ring which is a subring of $a$ ring $\mathfrak{v}^{\prime}$ and let $b$ be an element of $\mathfrak{b}^{\prime}$. If there exists an element $a$ of $\mathfrak{o}$ which is not a zero-divisor in $\mathfrak{v}^{\prime}$ such that $a b^{n}$ is in $\mathfrak{o}$ for any natural number $n$, then $b$ is integral over $\mathfrak{v}$.

Proof. The ring $\mathfrak{o}[b]$ is contained in a finite $\mathfrak{o}$-module $\mathfrak{o}+\mathfrak{v} a^{-1}$. Since $\mathfrak{o}$ is Noetherian, $\mathfrak{v}[b]$ is a finite $\mathfrak{v}$-module, whence $b$ is integral over $\mathfrak{v}$.

\section{$\S 5$. Integral extensions.}

Let $\mathfrak{o}$ be an integrity domain. An over-ring $\mathfrak{o}^{\prime}$ of $\mathfrak{o}$ is called an integral extension of $\mathfrak{o}$ if $\mathfrak{v}^{\prime}$ is an integrity domain and if $\mathfrak{v}^{\prime}$ is integral over $\mathfrak{b}$. Here, if the field of quotients of $\mathfrak{v}^{\prime}$ is finite over that of $\mathfrak{o}$, then we say that $\mathfrak{v}^{\prime}$ is almost finite over $\mathfrak{o}$.

An integrity domain which is integrally closed in its field of quotients is called a normal ring. When $\mathfrak{v}$ is a normal ring, an integral extension $\mathfrak{v}^{\prime}$ of $\mathfrak{v}$ is called a normal extension of $\mathfrak{v}$ if $\mathfrak{v}^{\prime}$ is the integral closure of $\mathfrak{v}$ in a normal extension of the field of quotients of $\mathfrak{v}$ (in the sense of Galois theory (need not to be separable)).

Proposition 1. Let $\mathfrak{v}$ be a normal ring and let $\mathfrak{o}^{\prime}$ be a normal extension of $\mathfrak{b}$. Then for any prime ideal $\mathfrak{p}$ of $\mathfrak{o}$, the prime ideals of $\mathfrak{v}^{\prime}$ which lie over $\mathfrak{p}$ are conjugate to each other (that is, if prime 
ideals $\mathfrak{p}_{1}^{\prime}$ and $\mathfrak{p}_{2}^{\prime}$ lie over $\mathfrak{p}$, then there exists an automorphism of $\mathfrak{o}^{\prime}$ over o which mapps $\mathfrak{p}_{1}^{\prime}$ to $\mathfrak{p}_{2}^{\prime}{ }_{2}$ ). (Cf. [4] or [7])

Proof. We first assume that $\mathfrak{o}^{\prime}$ is almost finite over $\mathfrak{o}$. Assume that prime ideals $\mathfrak{p}_{1}^{\prime}$ and $\mathfrak{p}_{2}^{\prime}$ lie over $\mathfrak{p}$ and that $\mathfrak{p}_{1}^{\prime}$ is not conjugate to $\mathfrak{p}_{2}^{\prime}$. Let $\mathfrak{p}_{2}^{\prime}, \cdots, \mathfrak{p}_{n}^{\prime}$ be the set of all prime ideals of $\mathfrak{p}^{\prime}$ which are conjugate to $\mathfrak{p}_{2}^{\prime}$. Then $\mathfrak{p}_{i}^{\prime}$ lies over $\mathfrak{p}$ for each $i$. Therefore there is no inclusion relation among $\mathfrak{p}_{1}^{\prime}, \cdots, \mathfrak{p}_{n}^{\prime}$ by Corollary 1 to Proposition 1 in $\S 4$. Therefore there exists an element $a$ of $\mathfrak{p}_{1}^{\prime}$ which is in none of $\mathfrak{p}_{2}^{\prime}, \cdots, \mathfrak{p}_{n}^{\prime}$ by Proposition 4 in $\S 1$. Then every conjugate of $a$ is not in $p_{2}^{\prime}$, whence the norm of $a$ in $\mathfrak{v}$ is not in $p_{2}^{\prime}$ and therefore it is not in $\mathfrak{p}$. This is a contradiction because $\mathfrak{p}_{1}^{\prime} \cap \mathfrak{v}=\mathfrak{p}$. Thus we have proved our assertion in this case. Now the general case can be proved easily by Zorn's lemma.

Corollary. Let $\mathfrak{o}$ be a normal ring and let $\mathfrak{o}^{\prime}$ be an integral extension of $\mathfrak{o}$. Let $\mathfrak{p}_{1} \supset \mathfrak{p}_{2} \supset \cdots \supset \mathfrak{p}_{r}$ be a descending chain of prime ideals $\mathfrak{p}_{\mathfrak{i}}$ of $\mathfrak{v}$ and let $\mathfrak{p}_{1}^{\prime}$ be a prime ideal of $\mathfrak{v}^{\prime}$ which lies over $\mathfrak{p}_{1}$. Then there exists a descending chain of prime ideals $\mathfrak{p}_{1}^{\prime} \supset \mathfrak{p}_{2}^{\prime} \supset \cdots \supset \mathfrak{p}_{r}^{\prime}$ of $\mathfrak{o}^{\prime}$ such that each $\mathfrak{p}^{\prime}$ lies over $\mathfrak{p}$. (Krull [7], cf. [4])

Proof. Let $\mathfrak{b}^{\prime \prime}$ be a normal extension of $\mathfrak{o}$ containing $\mathfrak{b}^{\prime}$ and let $\mathfrak{p}^{\prime \prime}{ }_{1}$ be a prime ideal of $\mathfrak{b}^{\prime \prime}$ which lies over $\mathfrak{p}_{1}^{\prime}$. On the other hand let $\mathfrak{q}_{1}^{\prime \prime} \supset \mathfrak{q}_{2}^{\prime \prime} \supset \cdots \supset \mathfrak{q}^{\prime \prime}$, be a descending chain of prime ideals of $\mathfrak{g}^{\prime \prime}$ such that $\mathfrak{q}^{\prime \prime}{ }_{i} \cap \mathfrak{v}=\mathfrak{p}_{6}$ for each $i$; existence follows from Corollary 2 to Proposition 1 in $\S 4$. Take an automorphism $\sigma$ of $\mathfrak{o}^{\prime \prime}$ over o which mapps $\mathfrak{q}^{\prime \prime}{ }_{1}$ to $\mathfrak{p}^{\prime \prime}{ }_{1}$ and set $\mathfrak{p}^{\prime \prime}{ }_{i}=\mathfrak{q}^{\prime \prime}{ }_{i}, \mathfrak{p}_{i}^{\prime}=\mathfrak{p}^{\prime \prime}{ }_{i} \cap \mathfrak{o}^{\prime}$ for each $i$. Then each $\mathfrak{p}_{i}^{\prime}$ lies over $\mathfrak{p}_{i}$ and $\mathfrak{p}_{1}^{\prime} \supset \mathfrak{p}_{2}^{\prime} \supset \cdots \supset \mathfrak{p}^{\prime}$.

Proposition 2. Let o be a normal ring and let $f(x)$ be a monic polynomial over $\mathfrak{o}$ in an indeterminate $x$. Set $\mathfrak{b}=\mathfrak{o}[x] /(f(x))$ and let $d$ be the discriminant of $f(x)$. If $\mathrm{D}^{\prime}$ is the integral closure of $\mathrm{D}$ in its total quotient ring, then d $\mathrm{d}^{\prime}$ is contained in $\mathfrak{b}$. (Zariski [12]).

Proof. If $d=0$, our assertion is trivial. Therefore we assume that $d \neq 0$. Let $a$ be the residue class of $x$ in $\delta$. Let $k$ be the field of quotients of $\mathfrak{o}$ and let $L$ be a field containing $k$ and all roots of $f(x)$. For each root $a_{t}$ of $f(x)$, there exists a homomorphism $\phi_{i}$ from $\mathfrak{D}^{\prime}$ into $L$ which mapps $a$ to $a_{i}$. Now let $b$ be an arbitrary element of $\mathrm{b}^{\prime}$. Then $b=\sum_{j=0}^{n-1} u_{j} a^{j}\left(u_{j} \in k, n=\right.$ degree of $f(x))$. Then $\phi_{i}(b)=\sum_{j} u_{j} a_{i}^{j}$ and we consider these equalities as linear equations in the unknown $u_{j}$ 's. The determinant $D$ of these equations is $\pm I_{i<j}\left(a_{i}-a_{j}\right)$. Therefore $D^{2}=d$. Since $\phi_{i}(b)$ 
and $a_{\mathfrak{t}}$ are integral over $\mathfrak{o}, d u_{j}^{\prime} \mathrm{s}$ are integral over $\mathfrak{o}$. Since $d u_{j}$ 's are in $k$ and since $\mathfrak{o}$ is normal, we have $d u_{j}$ 's are in $\mathfrak{o}$. Therefore $d b$ is in $\mathfrak{b}$, whence $d \mathfrak{b}^{\prime}$ is contained in $\mathfrak{b}$.

Corollary 1. Let $\mathfrak{o}, f(x)$ and $d$ be the same as in above proposition. Assume that $\mathfrak{a}$ is an ideal of $\mathfrak{v}[x]$ which contains $f(x)$ and whose prime divisors meet $\mathfrak{o}$ only with zero. Set $\mathfrak{B}=\mathfrak{o} / \mathfrak{a}$ and let $\mathfrak{S}^{\prime}$ be the integral closure of $\mathfrak{s}$ in its total quotient ring. Then $d \mathfrak{s}^{\prime}$ is contained in $\mathfrak{s}^{*}$ )

Proof. If $d=0$, our assertion is trivial. Therefore we assume that $d \neq 0$. Then the ideal $f(x) \mathfrak{D}[x]$ is semi-prime, hence the same is true of $\mathfrak{a}$ by our assumption. Therefore $\mathfrak{g}^{\prime}$ is a direct summand of $\mathfrak{b}^{\prime}$ in the proposition. Therefore we have $d \mathfrak{s}^{\prime}$ is contained in $\mathfrak{b}$.

Corollary 2. An almost finite separable integral extension of a Noetherian normal ring is a finite extension.

$\S 6$. The J-radical of a ring.

The intersection of all maximal ideals of a ring $\mathfrak{o}$ is called the $J$-radical of $\mathfrak{o}$. If an element $a$ of $\mathfrak{v}$ is congruent 1 modulo its $J$-radical, then $a$ is a unit in $\mathfrak{o}$.

Proposition 1. Let $M$ be a finite module over a ring $\mathfrak{o}$. Assume that an ideal $\mathfrak{a}$ of $\mathfrak{d}$ has the following properties: 1) $\mathrm{a} M=M$ and 2) if $a m=0 \quad(a \in \mathfrak{0}, m \in M)$ and if $a-1$ is in $a$, then $m=0$. Then we have $M=0$.

Proof. Let $u_{1}, \cdots, u_{n}$ be elements of $M$ such that $M=\sum_{i} \mathrm{D} u_{i}$. Since $\mathrm{a} M=M$, there exist elements $a_{i j}$ of a such that $u_{i}=\sum_{j} a_{i j} u_{j}$. Let $d$ be the determinant $\left|\left(\delta_{i j}-a_{i j}\right)\right|$ (where $\delta_{i j}$ are Kronecker's $\delta)$. Then $d u_{i}=0$ for all $i$. Since $d-1$ is in $\mathfrak{n}$, we have $u_{i}=0$ for all $i$, which shows that $M=0$.

Corollary 1. Let $M$ be a finite module over a ring $\mathfrak{v}$ and let $\mathfrak{m}$ be the J-radical of $\mathfrak{o}$. If $\mathfrak{m} M=M$, then $M=0$.

Corollary 2. Let $M$ be a finite module over a ring $\mathfrak{D}$ and let $\mathrm{m}$ be the J-radical of $\mathrm{o}$. If $N$ is a submodule of $M$ such that $M=\mathrm{m} M+N$, then $M=N$. (Azumaya's lemma ; see [8])

Proof. Set $M^{\prime}=M / N$. Then $M^{\prime}$ is a finite o-module and $\mathrm{m} M^{\prime}=M^{\prime}$. Therefore $M^{\prime}=0$, whence $M=N$.

Proposition 2. Let $\mathfrak{d}$ be a Noetherian ring and let $\mathfrak{a}$ be its ideal. Then ${ }_{n=1}^{\infty} \mathfrak{a}^{n}=0$ if and only if every element $a$ of $\mathfrak{v}$ such that

* It will be easy to see that $a$ is generated by a monic polynomial which is a factor of $f(x)$. 
$a-1$ is in $\mathfrak{a}$ is not a zero-divisor. (Chevalley [3], Krull [5], [7])

Proof. Assume that an element $a$ of $\mathfrak{o}$ such that $a-1$ is in $\mathfrak{a}$ is a zero-divisor. Let $b$ be an element of $\mathfrak{o}$ such that $a b=0 \quad(b \neq 0)$. Then $b=b(a-1)^{n}$ for any $n$, whence $b$ is in $\cap a^{n}$. Therefore $\cap \mathfrak{a}^{n} \neq 0$. Conversely, assume that there is no such $\stackrel{n}{a}$. Set $\mathfrak{n}=\cap \mathfrak{a}^{n}$. Then by Proposition 1 , we have only to show that an $\supseteq \mathfrak{n}$. Take primary ideals $\mathfrak{q}_{1}, \cdots, \mathfrak{q}_{r}$ of $\mathfrak{o}$ such that $\mathfrak{a} \mathfrak{n}=\mathfrak{q}_{1} \cap \cdots \cap \mathfrak{q}_{r}$ and let $\mathfrak{p}_{1}$, $\cdots, \mathfrak{p}_{r}$ be prime divisors of $\mathfrak{q}_{1}, \cdots, \mathfrak{q}_{r}$ respectively. For $\mathfrak{p}_{i}$ such that $\mathfrak{a} \nsubseteq \mathfrak{p}_{i}$, since $\mathfrak{a n} \subseteq \mathfrak{q}_{i}$ and since $\mathfrak{q}_{i}$ is a primary ideal belonging to $\mathfrak{p}_{i}$, we have $\mathfrak{n} \subseteq \mathfrak{q}_{i}$; for $\mathfrak{p}_{i}$ such that $\mathfrak{a} \subseteq \mathfrak{p}_{i}$, since $\mathfrak{q}_{i}$ is a strongly primary ideal belonging to $\mathfrak{p}_{i}$, a power of $\mathfrak{p}_{i}$ is in $\mathfrak{q}_{i}$, whence $\mathfrak{n} \subseteq \mathfrak{q}_{i}$. Therefore every $\mathfrak{q}_{i}$ contains $\mathfrak{n}$, which shows that $\mathfrak{n} \subseteq \mathfrak{a n}$.

Corollary. If $\mathrm{m}$ is the J-radical of a Noetherian ring, then $\cap \mathfrak{m}^{n}=0$.

Proposition 3. Let a be a non-unit of a Noetherian ring $\mathfrak{v}$. If either $a$ is in the J-radical of $\mathfrak{v}$ or $\mathfrak{v}$ is an integrity domain, then av cannot contain properly any prime ideal other than zero.

Proof. Assume that $a_{\mathfrak{o}}$ contains a prime ideal $\mathfrak{p}$ properly. Then for any element $c$ of $\mathfrak{p}$, there exists an element $c^{\prime}$ of $\mathfrak{o}$ such that $c=a c^{\prime}$. Since $a$ is not in $\mathfrak{p}$, we have $c^{\prime}$ is in $\mathfrak{p}$. Therefore $\mathfrak{p}=a \mathfrak{p}$. Then we see our assertion by Proposition 1 .

\section{$\S 7$. The minimum condition.}

Proposition 1. Let a be an ideal of a ring o. Assume that 1) $\mathfrak{a}$ is contained in the radical of $\mathfrak{o}$ and 2) the minimum condition holds for ideals contained in a. Then a is nilpotent. (Asano [2])

Pronf. It is sufficient to show that $\mathfrak{a}^{2} \neq \mathfrak{a}$ if $\mathfrak{a} \neq 0$. Assume that $\mathfrak{a}^{2}=\mathfrak{a}(\mathfrak{a} \neq 0)$. Let $\mathfrak{b}$ be an ideal of $\mathfrak{v}$ contained in $\mathfrak{a}$ which is minimal among ideals whose product with $\mathfrak{a}$ are different from 0 . Let $\mathfrak{p}$ be the set of elements $x$ of $\mathfrak{p}$ such that $\mathfrak{b} x \mathfrak{a}=0$. Then $\mathfrak{p}$ is a prime ideal; for if $c d \epsilon \mathfrak{p}, c \notin \mathfrak{p}$, then by the minimality of $\mathfrak{b}$, we have $\mathfrak{b} c=\mathfrak{b}$, whence $\mathfrak{b} d \mathfrak{a}=0$ and therefore $d$ is in $\mathfrak{p}$. Since a is in the radical of $\mathfrak{b}, \mathfrak{p}$ contains $\mathfrak{a}$, whence $\mathfrak{b} \mathfrak{a}^{\mathfrak{a}}=\mathfrak{b} \mathfrak{a}=0$, which is a contradiction.

Lemma 1. Let $\mathfrak{o}_{1}, \cdots, \mathfrak{v}_{\imath}$ be rings and let $\mathfrak{v}$ be the direct sum of them. Then the minimum condition for ideals holds in $\mathfrak{v}$ if and only if it holds in every $\mathfrak{o}_{i}$.

The proof is easy.

Lamma 2. Let $k$ be a field and let $M$ be a $k$-module. Then 
the minimum condition for submodulei holds in $M$ if and only if $M$ is a finite $k$-module; in this case, $M$ has a decomposition series.

The proof is easy.

Proposition 2. Let $\mathfrak{v}$ be a ring. Then the minimum condition for ideals holds in $\mathfrak{o}$ if and only if 1) $\mathfrak{o}$ is Noetherian and 2) every prime ideal of $\mathfrak{o}$ is maximal. (Akizuki [1])

Proof. We first assume that $\mathfrak{o}$ is Noetherian and that every prime ideal is maximal. Then every prime ideal of $\mathfrak{p}$ is a prime divisor of zero ideal. Therefore $\mathfrak{v}$ has only a finite number of prime ideals, say $p_{1}, \cdots, \mathfrak{p}_{r}$. Then $\mathfrak{p}_{1} \cap \cdots \cap \mathfrak{p}_{r}$ is the radical of $\mathfrak{v}$. Since $\mathfrak{o}$ is Noetherian, there exists a natural number $n$ such that $\left(p_{1} \cap \cdots \cap p_{r}\right)^{n}=0$. Since each $\mathfrak{p}_{i}$ is maximal, $\mathfrak{p}_{i}^{n}+\mathfrak{p}_{j}^{n}=\mathfrak{0}$ if $i \neq j$. Therefore $\mathfrak{v}$ is isomorphic to the direct sum of rings $\mathfrak{v} / \mathfrak{p}_{t}^{n}(i=1, \cdots, r)$. Therefore, by Lemma 1 , we may assume that $\mathfrak{p}_{1}$ is the unique maximal ideal of $\mathfrak{v}$. Then each $\mathfrak{p}_{1}{ }^{j} / \mathfrak{p}_{1}{ }^{j+1}$ is a finite $\mathfrak{o} / \mathfrak{p}_{1}$-module and $\mathfrak{p}_{1}{ }^{n}=0$. Since $\mathfrak{o} / \mathfrak{p}_{1}$ is a field, each $\mathfrak{p}_{1}{ }^{j} / \mathfrak{p}_{1}{ }^{j+1}$ has a decomposition series, whence $\mathfrak{v}$ has a decomposition series. Therefore the minimum condition for ideals holds in $\mathfrak{v}$ by virtue of the JordanHölder-Schreier theorem. Conversely, assume that the minimum condition holds in $\mathfrak{v}$. Let $\mathfrak{p}$ be a prime ideal of $\mathfrak{v}$. Then the minimum condition holds in $\mathfrak{o} / \mathfrak{p}$. Since $\mathfrak{o} / \mathfrak{p}$ is an integrity domain, $\mathfrak{p}$ must be a maximal ideal. If $p_{1}, \cdots, p_{m}$ are maximal ideals, each of which is different from another, then $\mathfrak{p}_{1} \cap \cdots \cap \mathfrak{p}_{m} \neq \mathfrak{p}_{1} \cap \cdots \cap \mathfrak{p}_{m-1}$. Therefore $\mathfrak{v}$ has only a finite number of maximal ideals, say $\mathfrak{p}_{1}, \cdots, \mathfrak{p}_{r}$. Then by Proposition $1, \quad\left(\mathfrak{p}_{1} \cap \cdots \cap \mathfrak{p}_{2}\right)^{n}=0$ for some $n$. Therefore $\mathfrak{o}$ is isomorphic to the direct sum of 1 ings $\mathfrak{o} / \mathfrak{p}_{i}^{n} \quad(i=1$, $\cdots, r)$. Therefore we may assume that $\mathfrak{p}=\mathfrak{p}_{1}$ is the unique maximal ideal of $\mathfrak{o}$. Set $\mathfrak{a}_{i}=0: \mathfrak{p}^{i}\left(=\mathfrak{a}_{i-1}: \mathfrak{p}\right.$ if $\left.i \geq 2\right)$. Then $\mathfrak{a}_{1}$ is an $\mathfrak{o} / \mathfrak{p}$-module and every submodule of $\mathfrak{a}_{1}$ is an ideal of $\mathfrak{o}$, whence the minimum condition for submodulei holds in $a_{1}$. Therefore $a_{1}$ is a finite $\mathfrak{o} / \mathfrak{p}$-module. Similarly, each $\mathfrak{a}_{i} / \mathfrak{a}_{i-1}$ is a finite $\mathfrak{o} / \mathfrak{p}$-module Therefore $\mathfrak{v}$ has a decomposition series, whence $\mathfrak{o}$ is Noetherian.

Remark. When we consider rings without identity, the same holds under the assumption that for any ideal there exists a maximal prime ideal which contains the given ideal. (The proof is the same.) This assumption is essential; for if we omit this assumption, there exists a ring which satisfies the minimum condition and which does not satisfy the maximum condition. Observe 
further that when the identity is added to a ring without identity, the new ring may not satisfy the minimum condition.

\section{$\S 8$. Rank of ideals.}

We say that a ring $\mathfrak{o}$ is of rank $r$ if there exists a chain $\mathfrak{p}_{0} \supset \mathfrak{p}_{1} \supset \cdots \supset \mathfrak{p}_{\text {. }}$ of prime ideals $\mathfrak{p}_{i}$ of $\mathfrak{p}$ and if there exists no such chain with more terms. ${ }^{*}$ ) If there exists no such $r$, then we say that $\mathfrak{v}$ is of rank infinite.

For a prime ideal $\mathfrak{p}$ of a ring $\mathfrak{o}$, the rank of $\mathfrak{b}_{\mathfrak{p}}$ is called the rank of $\mathfrak{p}$; for an arbitrary ideal $\mathfrak{a}$ of $\mathfrak{o}$, the minimum of rank of prime divisors of $\mathfrak{a}$ is called the rank of $\mathfrak{a}$. Further the rank of $\mathfrak{o} / \mathfrak{a}$ is called the co-rank of $a^{*}$ )

Proposition 1. If a ring $\mathfrak{v}^{\prime}$ is integral over its subring $\mathfrak{o}$, then rank $\mathfrak{b}^{\prime}=$ rank o. $\quad$ (Krull [7]; cf. [4])

This follows immediately from Corollary 2 to Proposition 1 in $\S 4$.

Proposition 2. Assume that $\mathfrak{v}^{\prime}$ is an integral extension of a normal ring $\mathfrak{o}$. If $\mathfrak{a}^{\prime}$ is an ideal of $\mathfrak{o}^{\prime}$, then rank $\mathfrak{a}^{\prime}=\operatorname{rank}\left(\mathfrak{a}^{\prime} \cap \mathfrak{o}\right)$.

Proof. When $\mathfrak{a}^{\prime}$ is a prime ideal, our assertion follows from the corollary to Proposition 1 in $\S 5$. Now let $\mathfrak{p}^{\prime}$ be a minimal prime divisor of $\mathfrak{a}^{\prime}$ such that rank $\mathfrak{a}^{\prime}=$ rank $\mathfrak{p}^{\prime}$. Then $\mathfrak{p}^{\prime} \cap \mathfrak{v}$ contains $\mathfrak{a}^{\prime} \cap \mathfrak{v}$, whence rank $\left(\mathfrak{a}^{\prime} \cap \mathfrak{o}\right) \leq$ rank $\mathfrak{a}^{\prime}$. Conversely, let $\mathfrak{p}$ be a minimal prime divisor of $\mathfrak{a}^{\prime} \cap \mathfrak{o}$ such that rank $\mathfrak{p}=\operatorname{rank}\left(\mathfrak{a}^{\prime} \cap \mathfrak{o}\right)$. Since $\mathfrak{v}^{\prime} / \mathfrak{a}^{\prime}$ is integral over $\mathfrak{v} /\left(\mathfrak{a}^{\prime} \cap \mathfrak{v}\right)$, there exists a prime ideal $\mathfrak{p}^{\prime}$ of $\mathfrak{v}^{\prime}$ which lies over $\mathfrak{p}$ and contains $\mathfrak{a}^{\prime}$. Then rank $\left(\mathfrak{a}^{\prime} \cap \mathfrak{o}\right)=$ rank $\mathfrak{p}=$ rand $\mathfrak{p}^{\prime} \geq$ rank $\mathfrak{a}^{\prime}$. Therefore we have rank $\mathfrak{a}^{\prime}=$ rank $\left(\mathfrak{a}^{\prime} \cap \mathfrak{o}\right)$.

Lemma 1. Let $\mathfrak{o}$ be a Noetherian integrity domain and let $a$ be a non-unit in $\mathfrak{v}$ which is not zero. If $\mathfrak{p}$ is a minimal prime divisor of $a \mathfrak{0}$, then $\mathfrak{p}$ is of rank 1. (Krull [5])

Proof. Considering $\mathfrak{o p}$ instead of $\mathfrak{p}$, we may assume that $\mathfrak{p}$ is the unique maximal ideal of $\mathfrak{b}$. Let $\mathfrak{q}$ be a prime ideal of $\mathfrak{o}$ different from $\mathfrak{p}$. Set $\mathfrak{q}^{(i)}=\mathfrak{q}^{i} \mathfrak{o}_{\mathfrak{q}} \cap \mathfrak{v}$ (which is called the $i$-th formal power of $q$ ). Since $\cap_{i} q^{i} \mathfrak{o}_{q}=0$ by Proposition 2 in $\S 6, \cap_{i} q^{(i)}=0$. Set $\mathfrak{a}_{i}=\mathfrak{q}^{(i)}+a_{\mathfrak{o}}$. Then there exists a number $n$ such that $\mathfrak{a}_{i}=\mathfrak{a}_{n}$ for any $i \geq n$, because in $\mathfrak{o} / a \mathfrak{o}$ the minimum condition for ideals

* In usual, these notions are called "dimension". But in connection with the notion of dimension in algebraic geometry, it seems to the writer that we had better to avoid the term "dimension" for these concepts. 
holds by Proposition 2 in $\S 7$. We have $\mathfrak{q}^{(n)} \subseteq \mathfrak{q}^{(i)}+a$ o. Set $\mathfrak{q}^{\prime}=$ $\mathfrak{q}^{(n)} / \mathfrak{q}^{(i)}, \mathfrak{v}^{\prime}=\mathfrak{v} / \mathfrak{q}^{(i)}$ and $a^{\prime}=$ residue class of $a$ in $\mathfrak{v}^{\prime}$. Then since $a$ is not in $\mathfrak{q}$ and since $\mathfrak{q}^{\prime}$ is a primary ideal belonging to $\mathfrak{q} / \mathfrak{q}^{(i)}, \mathfrak{q}^{\prime} \subseteq a^{\prime} \mathfrak{v}^{\prime}$ shows that $\mathfrak{q}^{\prime}=a^{\prime} \mathfrak{q}^{\prime}$, whence $\mathfrak{q}^{\prime}=0$ because $a^{\prime}$ is in the J-radical of $\mathfrak{v}^{\prime}$. Therefore we have $\mathfrak{q}^{(i)}=\mathfrak{q}^{(n)}$ and $\cap_{i} \mathfrak{q}^{(i)}=\mathfrak{q}^{(n)}=0$, which shows that $\mathfrak{q}=0$, because $\mathfrak{v}$ is an integrity domain.

Proposition 3. Let $\mathfrak{a}$ be an ideal of a Noetherian ring $\mathfrak{0}$. Assume that $\mathfrak{a}$ is generated by $r$ elements. Then for any minimal prime divisor $\mathfrak{p}$ of $\mathfrak{a}$, rank $\mathfrak{p}$ is not greater than $r$. (Krull [5])

Proof. Let $\mathfrak{p}=\mathfrak{p}_{0} \supset \mathfrak{p}_{1} \supset \cdots \supset \mathfrak{p}_{s}$ be a chain of prime ideals $\mathfrak{p}_{s}$ such that $\mathfrak{p}_{0} / \mathfrak{p}_{1}$ is of rank 1 . Then we have only to show that $s \leq r$. Then considering $\mathfrak{o} / \mathfrak{p}_{\mathrm{s}}$ instead of $\mathfrak{o}$, we may assume that $\mathfrak{o}$ is an integrity domain. Therefore, if $r=1$, our assertion follows from Lemma 1. Therefore we prove our assertion by induction on $r$. Considering $\mathfrak{o}_{\mathfrak{p}}$ instead of $\mathfrak{d}$, we may assume that $\mathfrak{p}$ is the unique maximal ideal of $\mathfrak{o}$; in this case, $\mathfrak{a}$ is a primary ideal belonging to $\mathfrak{p}$. Let $a_{1}, \cdots, a_{r}$ be elements of a which generate $\mathfrak{a}$. We may assume that $a_{1}$ is not in $\mathfrak{p}_{1}$. Then $p_{1}+a_{1} \mathfrak{o}$ is a primary ideal belonging to $\mathfrak{p}$, whence there exists a natural number $t$ such that $a_{i}{ }^{t}$ is in $p_{1}+a_{1} \mathrm{o}$ for every $i$; we write $a_{i}^{t}=a_{1} b_{t}+c_{t}$ with elements $b_{i}$ of $\mathfrak{v}$ and $c_{i}$ of $\mathfrak{p}_{1}$. Set $\mathfrak{a}^{\prime}=\sum_{t=2}^{r} c_{i} \mathrm{o}$. Then since $a_{i}^{t}$ is in $\mathfrak{a}^{\prime}+a_{1} \mathfrak{v}, \mathfrak{a}^{\prime}+a_{1} \mathfrak{v}$ is a primary ideal belonging to $\mathfrak{p}$. Let $\mathfrak{p}^{\prime}$ be a minimal prime divisor of $\mathfrak{a}^{\prime}$ which is contained in $\mathfrak{p}_{1}$. Then since $\mathfrak{p}^{\prime}+a_{1} \mathfrak{v}$ is a primary ideal belonging to $\mathfrak{p}, \mathfrak{p} / \mathfrak{p}^{\prime}$ is of rank 1 , whence $\mathfrak{p}^{\prime}=\mathfrak{p}_{1}$. Since $\mathfrak{a}^{\prime}$ is generated by $r-1$ elements, we have rank $\mathfrak{p}_{1}$ is not greater than $r-1$ by our induction assumption. Therefore $s \leq r$ and our proof is completed.

Corollary. Rank of an ideal in a Noetherian ring is finite.

Proposition 4. If $\mathfrak{a}$ is an ideal of a Noetherian ring $\mathfrak{o}$ and if $\mathfrak{a}$ is of rank $r$, then there exist elements $a_{1}, \cdots, a_{r}$ of a such that $\sum_{i=1}^{s} a_{i} \mathfrak{0}$ is of rank $s$ for any $s \leq r$. (We regard that the ideal generated by the empty set is zero.)

Proof. When $s=0$, our assertion is evident. Therefore we prove our assertion by induction on $s$; we assume that there are $s-1$ elements $a_{1}, \cdots, a_{s-1}$ of $\mathfrak{a}$ such that rank $\left(\sum_{i=1}^{t} a_{i} \mathfrak{v}\right)=t$ for any $t \leq s-1$. Let $\mathfrak{p}_{1}, \cdots, \mathfrak{p}_{n}$ be prime divisors of $\sum_{i=1}^{d-1} a_{i} \mathfrak{D}$ whose rank are $s-1$. If $s-1<r$, then $a$ is not contained in any of $p_{i}^{\prime}$ 's, whence there exists an element $a_{s}$ of $a$ which is not contained in any of $p_{i}$ 's by Proposition 4 in $\S 1$. Then the rank of $\sum_{i=1}^{s} a_{i} \mathfrak{0}$ is 
at least $s$, whence it is $s$ by Proposition 3 .

\section{$\S 9$. Normal Noetherian rings.}

A ring $\mathfrak{v}$ is called a Dedekind domain if it is Noetherian normal ring of rank 1 . Noetherian local ring is a Noetherian ring which has only one maximal ideal; Noetherian semi-local ring is a Noetherian ring which has only a finite number of maximal ideals. According to these notions, we use terminology such that local Dedekind domain and semi-local Dedekind domain.

Lemma 1. Let $\mathfrak{o}$ be a Noetherian local integrity domain with maximal ideal $\mathfrak{p}$. Let $a(\neq 0)$ be an element of $\mathfrak{p}$ and let $b$ be an element of $a_{0}: \mathfrak{p}$. When $\mathfrak{o}$ is of rank 1 , we assume that $a$ is irreducible (that is, $a$ cannot be expressed as the product of two elements of $\mathfrak{p})$ and that $a \mathfrak{v}: \mathfrak{p} \neq \mathfrak{v}$. Then $b / a$ is integral over $\mathfrak{o}$.

Proof. Let $h$ be an element of $\mathfrak{p}$ and set $c=(b / a) h$. Since $b$ is in $a_{\mathfrak{0}}: \mathfrak{p}, b h$ is in $a \mathfrak{v}$, whence $c$ is in $\mathfrak{o}$. Assume that $c$ is not in $\mathfrak{p}$. Then $a \mathfrak{v}=b h \mathfrak{v}$. Therefore, when $\mathfrak{v}$ is of rank 1 , this is a contradiction to our assumption that $a$ is irreducible and $b$ is in $\mathfrak{p}$. When $\mathfrak{v}$ is not of rank 1 , let $\mathfrak{p}^{\prime}$ be a minimal prime divisor of $h \mathfrak{v}$. Then $a$ is in $\mathfrak{p}^{\prime}$ because $a \mathfrak{v}=b h \mathfrak{v}$. Let $\mathfrak{q}^{\prime}$ be the primary component of $a \mathfrak{v}$ belonging to $\mathfrak{p}^{\prime}$. Since $\mathfrak{p}^{\prime} \neq \mathfrak{p}, \mathfrak{q}^{\prime}: \mathfrak{p}=\mathfrak{q}^{\prime}$, whence $b$ is in $\mathfrak{q}^{\prime}$. Then $a \mathfrak{v}=b h_{\mathfrak{o}}$ shows that $\mathfrak{q}^{\prime} \mathfrak{b}_{\mathfrak{p}^{\prime}} \subseteq \mathfrak{q}^{\prime} \mathfrak{p}^{\prime} \mathfrak{b}_{\mathfrak{p}^{\prime}}$ and therefore $\mathfrak{q}^{\prime} \mathfrak{b}_{\mathfrak{p}^{\prime}}=\mathfrak{q}^{\prime} \mathfrak{p}^{\prime} \mathfrak{b}_{\mathfrak{p}^{\prime}}$, which is a contradiction by Corollary 1 to Proposition 1 in $\S 6$ because $\mathfrak{p}^{\prime} \mathfrak{b}_{\mathfrak{p}^{\prime}}$ is the $J$-radical of $\mathfrak{o}_{\mathfrak{p}^{\prime}}$. Thus we see that $c$ is in $\mathfrak{p}$ in either case. Therefore $(b / a)^{n} h$ is in $\mathfrak{p}$ for every $n$. Therefore $b / a$ is integral over $\mathfrak{o}$ by Corollary 3 to Proposition 2 in $\S 4$.

Proposition 1. A semi-local Dedekind domain is a principal ideal ring. (Cf. [6])

Proof. Let first $\mathfrak{o}$ be a local Dedekind domain and let $a$ be an irreducible element of $\mathfrak{o}$. Then by Lemma $1, a \mathfrak{v}: \mathfrak{p}=\mathfrak{v}$, whence $a \mathfrak{v}=\mathfrak{p}$. Now let $\mathfrak{D}$ be a semi-local Dedekind domain with maximal ideals $\mathfrak{p}_{1}, \cdots, \mathfrak{p}_{n}$. Let $\mathfrak{m}$ be the $J$-radical of $\mathfrak{D}\left(\mathfrak{m}=\mathfrak{p}_{1} \cap \cdots \cap \mathfrak{p}_{n}\right)$. Then $\mathfrak{D} / \mathfrak{m}^{2}$ is isomorphic to the direct sum of $\mathfrak{D} / \mathfrak{h}_{1}{ }^{2}, \cdots, \mathfrak{b} / \mathfrak{p}^{2}{ }^{2}$. Since $\mathfrak{D} / \mathfrak{p}_{i}{ }^{2}=\mathfrak{D}_{\mathfrak{p}_{i}} / \mathfrak{p}_{i}{ }^{\prime} \mathfrak{D}_{\mathfrak{p}_{i}}$ and since $\mathfrak{D}_{\mathfrak{p}_{i}}$ is a local Dedekind domain, we see that $\mathfrak{p}_{i} / \mathfrak{m}^{2}$ is generated by an element $a_{i}$. Let $a_{t}$ be an element of $\mathfrak{p}_{i}$ whose residue class is $a_{i}^{\prime}$. Then $\mathfrak{p}_{i}=a_{i} \mathfrak{0}+\mathfrak{m}^{2}$, whence $\mathfrak{p}_{i}=$ $a_{i} \mathfrak{v}+\mathfrak{p}_{i} \mathfrak{m}$. Therefore by Corollary 2 Proposition 1 in $\$ 6$, we have $\mathfrak{p}_{i}=a_{i} \mathrm{D}$. Since every maximal ideal of $\mathrm{D}$ is principal, every ideal of $\mathfrak{d}$ is principal (it is generated by an element of the form $a_{1}{ }^{\alpha_{1}}$ 
$\cdots \boldsymbol{a}_{n}^{\alpha_{n}}$, except for 0 ).

Corollary. A local Dedekind domain is a discrete valuation ring (and conversely).

Proposition 2. Let $\mathfrak{o}$ be a Noetherian integrity domain and let $a(\neq 0)$ be a non-unit in $\mathfrak{0}$. If av has imbedded prime divisors, then there exists an element $b$ of o such that $b / a$ is integral over $\mathfrak{o}$ and $b / a$ is not in $\mathrm{v}$. Conversely, if there exists such an element $b$ as above, then either there exists a minimal prime divisor $\mathfrak{p}$ of a such that $\mathfrak{o}_{\mathfrak{p}}$ is not normal or ao has imbedded prime divisors.

Proof. Assume that $a_{0}$ has an imbedded prime divisor q. Let $b^{\prime}$ be an element of $a_{\mathfrak{D}_{\mathfrak{q}}}: \mathfrak{q}_{\mathfrak{v}_{\mathrm{q}}}$ which is not in $a_{\mathfrak{v}_{\mathrm{q}}}$; existence of such $b^{\prime}$ follows from the expression of $a_{\mathfrak{o}_{\mathfrak{a}}}$ as the intersection of primary ideals. Then by Lemma $1, b^{\prime} / a$ is integral over $\mathfrak{o}_{q}$. Let $c_{1} / s_{1}, \cdots$, $c_{n} / s_{n}\left(c_{i}, s_{i} \in \mathfrak{D}, s_{i} \notin \mathfrak{q}\right)$ be elements of $\mathfrak{o}_{\mathfrak{q}}$ such that $\left(b^{\prime} / a\right)^{n}+\left(c_{1} / s_{1}\right)$ $\left(b^{\prime} / a\right)^{n-1}+\cdots+\left(c_{n} / s_{n}\right)=0$. Set $b=b^{\prime} s s_{1}, \cdots s_{n}$, where $s$ is an element of $\mathfrak{v}$ which is not in $\mathfrak{q}$ such that $b^{\prime} s$ is in $\mathfrak{o}$. Then $b / a$ is integral over $\mathfrak{o}$. Since $b^{\prime} / a$ is not in $\mathfrak{o}_{\mathfrak{q}}, b / a$ is not in $\mathfrak{o}_{\mathfrak{q}}$ and therefore $b / a$ is not in $\mathfrak{o}$. Conversely, we assume that there exists an element $b$ of $\mathfrak{v}$ such that $b / a$ is integral over $\mathfrak{v}$ and $b / a$ is not in $\mathfrak{o}$. Assume further that for every minimal prime divisor $\mathfrak{p}$ of $a \mathfrak{v}, \mathfrak{p} p$ is normal and that $a_{0}$ has no imbedded prime divisor. Let $\mathfrak{p}_{1}, \cdots$, $\mathfrak{p}_{r}$ be the set of (minimal) prime divisors of $a \mathfrak{0}$ and let $\mathfrak{q}_{1}, \cdots, \mathfrak{q}_{r}$ be the primary components of $a_{\mathfrak{o}}$ belonging to $\mathfrak{p}_{1}, \cdots, \mathfrak{p}_{r}$ respectively. Then since $a v$ has no imbedded prime divisor, $\mathfrak{q}_{1} \cap \cdots \cap q_{r}$ $=a \mathfrak{v}$. Since $b / a$ is integral over $\mathfrak{o}, b / a$ is integral over $\mathfrak{o}_{\mathfrak{p}}$, , whence $b / a$ is in $\mathfrak{o}_{\mathfrak{p}_{i}}$ (for each i). Therefore $b \mathfrak{o}_{\mathfrak{p}_{i}} \subseteq a \mathfrak{o p}_{\mathfrak{p}_{i}}=\mathfrak{q}_{i} \mathfrak{o p}_{\mathfrak{p}_{i}}$ and therefore $b \in \mathfrak{q}_{i} \mathfrak{o p}_{i} \cap \mathfrak{o}=\mathfrak{q}_{i}$ for each $i$, which shows that $b$ is in $a \mathfrak{v}$. This is a contradiction to our assumption that $b / a$ is not in $\mathfrak{o}$. Thus the proof is completed.

Corollary 1. A Noetherian integrity domain $\mathfrak{v}$ is normal if and only if the following two conditions are satisfied:

(1) For every prime ideal $p$ of rank $1, \mathfrak{o}_{\mathfrak{p}}$ is normal.

(2) Every principal ideal of o has no imbedded prime divisor. (Krull [6])

This follows from Proposition 2 and the following

Lemma 2. Any ring of quotients of a normal ring is also normal.

The proof is easy.

Corollary 2. A Noetherian integrity domain $\mathfrak{o}$ is normal if 
and only if every principal ideal of $\mathrm{o}$ is an intersection of formal powers of prime ideals of rank 1 (except for the zero ideal). (Krull [6])

This follows from Corollary 1 and the following

Lemma 3. Let $\mathfrak{o}$ be a Noetherian local integrity domain of rank 1. Then $\mathfrak{o}$ is normal if and only if $\mathfrak{p} / \mathfrak{p}^{2}$ is principal, where $\mathfrak{p}$ denotes the maximal ideal of $\mathfrak{o}$.

Proof. If $\mathfrak{o}$ is normal, then $\mathfrak{p}$ is principal, whence $\mathfrak{p} / \mathfrak{p}^{2}$ is principal. Conversely, if $\mathfrak{p} / \mathfrak{p}^{2}$ is principal, then $\mathfrak{p}$ is principal by Corollary 2 to Proposition 1 in $\S 6$. Therefore $\mathfrak{o}$ is a principal ideal ring and $\boldsymbol{o}$ is normal.

Corollary 3. A Noetherian normal ring $\mathfrak{o}$ is the intersection of all $\mathfrak{o p}$, where $\mathfrak{p}$ runs over all prime ideals of $\mathfrak{o}$ of rank 1 . (Krull [6])

Corollary 4. Let o be a Noetherian integrity domain. Assume that for every prime ideal $\mathfrak{p}$ of $\mathfrak{o}$ of rank $1, \mathfrak{o}_{\mathfrak{p}}$ is normal. If a principal ideal av $(a \in \mathfrak{p})$ has an imbedded prime divisor $\mathfrak{\mathfrak { q }}, \mathfrak{q}$ is also an imbedded prime divisor of bo for any non-zero element $b$ of $\mathfrak{q}$.

Proof. We may assume without loss of generality that $q$ is the unique maximal ideal of $\mathfrak{o}$. Let $d$ be an element of $a \mathfrak{o}: \mathfrak{p}$ which is not in $a_{\mathfrak{v}}$. Then $d / a$ is not in $\mathfrak{v}$ and is integral over $\mathfrak{v}$ by Lemma 1. Let $b$ be a non-zero element of $\mathfrak{q}$. Then $c=(d / a) b$ is in $\mathfrak{o}$. Since $d / a=c / b$, we see that $b \mathfrak{o}$ has $\mathfrak{q}$ as an imbedded prime divisor.

Remark. Let $\mathfrak{o}$ be an integrity domain (which may not be Noetherian). Then $\mathfrak{v}$ is the intersection of all $\mathfrak{o}_{\mathfrak{p}}$, where $\mathfrak{p}$ runs over all maximal ideals of $\mathfrak{b}$.

Proof. Let $\mathfrak{b}$ be the intersection of all $\mathfrak{o}_{\mathfrak{p}}$. Then $\mathfrak{o} \subseteq \mathfrak{D}$. Let $a$ be an element of $b$ and let $\mathfrak{a}$ be the set of elements of $\mathfrak{o}$ whose product with $a$ is in $\mathfrak{o}$. If $1 \in \mathfrak{a}$, then $a \in \mathfrak{p}$. We assume that $1 \notin \mathfrak{a}$. Since $\mathfrak{a}$ is an ideal of $\mathfrak{o}$, there exists a maximal ideal $\mathfrak{p}$ of $\mathfrak{v}$ which contains $\mathfrak{a}$. Then $\mathfrak{o}_{\mathfrak{p}}$ cannot contain $a$, which is a contradiction. Therefore $\mathfrak{d} \subseteq \mathfrak{v}$ and therefore $\mathfrak{d}=\mathfrak{b}$.

\section{BIBLIOGRAPHY}

1. Y. Akizuki, Teilerkettensatz und Vielfachenkettensatz, Proc. Phys.-Math. Soc. Japan (3) vol. 17 (193̄), pp. 337-345.

2. K. Asano, Theory of rings and ideals, Kindai Sûgaku Zensho (Kyôritsu) (1949) (in Japanese). 
3. C. Chevalley, On the theory of local rings, Ann. of Math. vol. 44 (1943), pp. 690-708.

4. I. S. Cohen- A. Seidenberg, Prime ideals and integral dependence, Bull. Amer. Math. Soc. vol. 52 (1946), pp. 252-261.

5. W. Krull, Primidealketten in allgemeinen Ringbereichen, S.-B. Heiderberg Akad. Wiss. vol. 7 (1928).

6. W. Krull, Idealtheorie, Ergeb. der Math. vol. 4 No. 3 (1935).

7. W. Krull, Beiträge zur Arithmetik kommutativer Integritätsbereiche, III, Math. Zeit. vol. 42 (1937), pp, 745-766.

8. M. Nagata, On the structure of complete local rings, Nagoya Math. Journ. vol. 1 (1950), pp. 63-70.

9. M. Nagata, On the theory of semi-local rings, Proc. Japan Acad. vol. 26 (1950), pp. 131-140.

10. A. I. Uzkov, On the rings of quotients of commutative rings, Mat. Sbornik N. S. 22 (64) (1948), pp. 439-441 (in Russian).

11. B. L. van der Waerden, Moderne Algebra, II, Grundl. Math. Wiss. in Einzeldarst. vol. 34 (1931).

12. O. Zariski, Sur la normalité analytique des variété normales, Ann. Inst. Fourier vol. 2 (1950), pp. 161-164. 